\title{
Analysis of possible anomalies in the QSO distribution of the Flesch \& Hardcastle catalogue
}

\author{
M. López-Corredoira ${ }^{1}$, C. M. Gutiérrez ${ }^{1}$, V. Mohan ${ }^{2}$, G. I. Gunthardt ${ }^{3,4}$, and M. S. Alonso ${ }^{5,6}$ \\ 1 Instituto de Astrofísica de Canarias, C/.Vía Láctea, s/n, 38200 La Laguna (S/C de Tenerife), Spain \\ e-mail: martinlc@iac.es \\ 2 Inter-University Centre for Astronomy and Astrophysics (IUCAA), Post Bag No. 4, Ganeshkhind, Pune 411 007, India \\ 3 Observatorio Astronómico, Universidad Nacional de Córdoba, Laprida 854, 5000 Córdoba, Argentina \\ 4 SECyT, Universidad Nacional de Córdoba, Argentina \\ 5 Consejo Nacional de Investigaciones Científicas y Técnicas, Argentina \\ ${ }^{6}$ Complejo Astronómico El Leoncito, Argentina
}

Received 26 June 2007 / Accepted 28 November 2007

ABSTRACT

\begin{abstract}
Aims. A recent catalogue by Flesch \& Hardcastle presents two major anomalies in the spatial distribution of QSO candidates: $i$ ) an apparent excess of such objects near bright galaxies, and ii) an excess of very bright QSO candidates compared to random background expectations in several regions of the sky. Because anyone of these anomalies would be relevant in a cosmological context, we carried out an extensive analysis of the probabilities quoted in that catalogue.

Methods. We determine the nature and redshift of a subsample of 30 sources in that catalogue by analysing their optical spectra (another 11 candidates were identified from existing public databases). These have allowed us to statistically check the reliability of the probabilities QSO status quoted by Flesch \& Hardcastle for their candidates.

Results. Only 12 of the 41 candidates turned out QSOs (7 of which have been identified here for the first time).

Conclusions. The probabilities of the QSOs' being the candidates given by Flesch \& Hardcastle are overestimated for $m_{B} \leq 17$ and for objects projected near $(\leq 1 \mathrm{arcmin})$ bright galaxies. This is the cause of the anomalies mentioned above.
\end{abstract}

Key words. quasars: general - galaxies: statistics - distance scale - catalogs

\section{Introduction}

At present, statistics on QSO distributions can be carried out using the recent public compilations of QSOs identified in the SDSS (Adelman-McCarthy et al. 2007) and 2dF (Croom et al. 2004) surveys. Furthermore, Flesch \& Hardcastle (2004, hereafter FH04) present an all-sky catalogue with 86009 optical counterparts of radio/X-ray sources as QSO candidates (with a probability greater than $40 \%$, according to $\mathrm{FH} 04$ ) that were not identified previously as such. This could be a useful catalogue to look for QSOs in regions that were not covered by SDSS or $2 \mathrm{dF}$, or for deeper magnitudes than their completeness limit. Nevertheless, since the FH04 catalogue does not give the identification of each source but just a statistical estimate of the probability that it be a QSO (or a star, a galaxy, or a wrongly identified optical counterpart of the X-ray/radio source), it must be used with care.

Flesch" claims that his catalogue "reveals a few galaxycentered fields which are populated by QSOs/candidates more thickly than expected by the background density of such QSOs," which would argue in favour of the hypothesis of QSOs with anomalous redshift and at the same distance as the galaxies with which they are associated (Arp 1998; Burbidge 2001). Because this is against one of the most basic assumptions in standard modern cosmology, we consider it interesting to check it on the basis of rigorous statistical computation. This is the main objective of this paper. In Sect. 2, we analyse in which cases (on

${ }^{1}$ See his web-page http://quasars .org/ the basis of the probabilities quoted by FH04) there are anomalies in the distribution of QSOs. By conducting optical spectroscopy (Sect. 3), we directly check the nature of a subsample of FH04 sources. On the basis of these observations, in Sect. 4 we check the reliability of the probabilities quoted by FH04. In Sect. 5 we reanalyse the distribution of QSOs and discuss the implications of our results.

\section{Anomalies in the distribution of QSO candidates}

\subsection{Exploring the whole sky}

The average number of QSOs up to $B$-magnitude $m_{B, 0}$ is (López-Corredoira \& Gutiérrez 2006a, Eq. (2)):

$$
\begin{aligned}
& N\left(m_{B}<m_{B, 0}\right)\left[\mathrm{deg}^{-2}\right]= \\
& \left\{\begin{array}{ll}
10^{-2.8+0.8\left(m_{B, 0}-15\right)}, & m_{B, 0} \leq 18.5 \\
1981.0-214.2 m_{b_{j}, 0}+5.792 m_{b_{j}, 0}^{2}, & m_{B, 0}>18.5
\end{array}\right\},
\end{aligned}
$$

where the conversion of the photographic to the Johnson bandpass is $m_{b_{j}, 0} \approx m_{B, 0}-0.14 \times\left(m_{B, 0}-m_{R, 0}\right)$.

Given a circular region of angular radius $R$ (deg.) with $n$ QSO candidates up to magnitude $m_{B, 0}$, the probability that FH04 expectations be compatible with the expectations for background QSOs is

$P=\sum_{m=0}^{n} P_{\mathrm{p}}(m) P_{\mathrm{q}}(m)$, 
where $P_{\mathrm{p}}(m)$ is the Poissonian probability of having $m$ sources in the circle

$P_{\mathrm{p}}(m)=\frac{\left[\pi R^{2} N\left(m_{B}<m_{B, 0}\right)\right]^{m} \mathrm{e}^{-\left[\pi R^{2} N\left(m_{B}<m_{B, 0}\right)\right]}}{m !}$,

and $P_{\mathrm{q}}(m)$ is the probability that we observe a number of $m$ QSOs among $n$ candidates given the a priori probabilities $p_{1}, \ldots, p_{n}$ :

$P_{\mathrm{q}}(m<n)=P_{\mathrm{q}}(n) \sum_{l_{1}, \ldots, l_{n-m}=1 ; l_{1}<\ldots<l_{n-m}}^{n}\left[\left(p_{l_{1}}^{-1}-1\right) \ldots\left(p_{l_{n-m}}^{-1}-1\right)\right]$,

$P_{\mathrm{q}}(n)=\prod_{i=1}^{n} p_{i}$

The probability of finding at least one region with low probability $P$ in the whole sky is

$P_{\text {sky }}=P \times\left(41252 \mathrm{deg}^{2}\right) \times \operatorname{Minimum}\left[\frac{1}{\pi R^{2}}, N\left(m_{B}<m_{B, 0}\right)\right]$.

We have explored the distribution of QSO candidates in several projected circles on the sky to check their compatibility with the expectation from background QSOs. In Table 1, we give the centre and radius of the regions and magnitudes that are very unlikely $\left(P_{\text {sky }}<0.05\right.$, i.e. $95 \%$ C.L. $)$ as a result of chance projection. The table shows many of these cases ${ }^{2}$.

The large excess of QSO candidates up to $B$-magnitude $\sim 19$ in the region centred at $\alpha \approx 84.9^{\circ}=5 \mathrm{~h} 39 \mathrm{~m} 36 \mathrm{~s}, \delta=-64^{\circ}$ and radius $0.4 \mathrm{deg}$ (26 QSO candidates in a $0.50 \mathrm{deg}^{2}$ region up to magnitude 19 while an average of $\sim 1$ QSO is expected as background) might be related with the Large Magellanic Clouds (LMC), whose centre is 7 degrees away. The region around LMC contains a number of X-ray sources (Pietsch \& Kahabka 1993; Haberl \& Pietsch 1999), many of them background QSOs (Kahabka 2002; Dobrzycki et al. 2005), but many of them are other kinds of objects that could have been confused with QSO candidates by FH04. Particularly, there is a very bright X-ray source exactly in this region: LMC X-3 (Haberl \& Pietsch 1999) with $\alpha=5 \mathrm{~h} 38 \mathrm{~m} 56.8 \mathrm{~s}, \delta=-64^{\circ} 05^{\prime} 12^{\prime \prime}$, a black hole binary, a Be star with a period 1.7 days. Most probably, the many ROSAT X-ray sources surrounding it might be attenuated reflections (by the X-ray detector, which might create ghost images when a strong source saturates it) of this very bright X-ray source.

However, the excess of very bright QSOs in many areas given in Table 1 cannot be explained by any similar contamination. For instance, in the circle centred at $\alpha \approx 69.282^{\circ}=$ $4 \mathrm{~h} 37 \mathrm{~m} 7.7 \mathrm{~s}, \delta=-30^{\circ}$ and radius $60 \mathrm{deg}$. (Twenty QSO candidates in a $1 / 4$ of the whole sky up to magnitude 14.5 , while an average of $\sim 7$ QSOs is expected as background.)

\subsection{Exploring the regions near bright galaxies}

Here we explore the regions near bright RC3 galaxies (Third Reference Catalog of Bright Galaxies; de Vaucouleurs et al. 1991, updated on 1995, February 16th). The RC3 catalogue is

\footnotetext{
2 These probabilities are conservative because: $i$ / the catalogue ignores QSO candidates with probabilities $\leq 40 \%$; ii/ it includes only those QSO candidates that were not classified previously; iii/it only includes QSOs that emit in radio or X-ray, which is not the whole QSO population; iv/ it is not complete for the faintest fluxes because the radio and X-ray catalogues used do not have the same depth all over the sky.
}

Table 1. Probabilities of QSO excesses.

\begin{tabular}{cccccc}
\hline \hline$\alpha\left({ }^{\circ}\right), \delta\left(^{\circ}\right)$ & $R\left(^{\circ}\right)$ & $m_{B, 0}$ & $n$ & $P$ & $P_{\text {sky }}$ \\
\hline $324.534,-66.000$ & 6 & 13.0 & 2 & 0.026 & 0.042 \\
$324.534,-66.000$ & 6 & 13.5 & 3 & 0.010 & 0.041 \\
$30.193,-58.000$ & 16 & 14.0 & 4 & 0.0041 & 0.042 \\
$58.476,-70.000$ & 20 & 13.0 & 2 & 0.021 & 0.035 \\
$59.006,-66.000$ & 24 & 13.0 & 2 & 0.023 & 0.038 \\
$62.229,-50.000$ & 40 & 13.0 & 3 & 0.021 & 0.035 \\
$62.229,-50.000$ & 40 & 14.0 & 6 & 0.0034 & 0.036 \\
$63.640,-45.000$ & 45 & 13.0 & 3 & 0.023 & 0.038 \\
$63.640,-45.000$ & 45 & 14.0 & 7 & 0.0021 & 0.022 \\
$63.640,-45.000$ & 45 & 14.5 & 12 & 0.0013 & 0.027 \\
$65.270,-40.000$ & 50 & 13.0 & 3 & 0.026 & 0.043 \\
$65.270,-40.000$ & 50 & 13.5 & 5 & 0.0071 & 0.029 \\
$65.270,-40.000$ & 50 & 14.0 & 8 & 0.0014 & 0.014 \\
$65.270,-40.000$ & 50 & 14.5 & 16 & $3.1 \times 10^{-4}$ & 0.0052 \\
$67.143,-35.000$ & 55 & 14.0 & 8 & 0.0041 & 0.043 \\
$67.143,-35.000$ & 55 & 14.5 & 16 & 0.0011 & 0.015 \\
$69.282,-30.000$ & 60 & 14.5 & 20 & $2.3 \times 10^{-4}$ & 0.0027 \\
$71.720,-25.000$ & 65 & 14.5 & 19 & $8.6 \times 10^{-4}$ & 0.0083 \\
$69.282,-30.000$ & 60 & 12.5 & 3 & 0.058 & 0.038 \\
$71.720,-25.000$ & 65 & 12.5 & 3 & 0.060 & 0.039 \\
$65.270,-40.000$ & 50 & 15.0 & 20 & 0.0020 & 0.033 \\
$67.143,-35.000$ & 55 & 15.0 & 22 & 0.0028 & 0.038 \\
$69.282,-30.000$ & 60 & 15.0 & 27 & $9.7 \times 10^{-4}$ & 0.011 \\
$71.720,-25.000$ & 65 & 15.0 & 27 & 0.0036 & 0.035 \\
$84.783,-64.200$ & 0.3 & 19.0 & 20 & $6.3 \times 10^{-8}$ & 0.0055 \\
$84.860,-64.000$ & 0.4 & 19.0 & 26 & $6.2 \times 10^{-9}$ & $5.4 \times 10^{-4}$ \\
$84.860,-64.000$ & 0.4 & 19.5 & 30 & $4.7 \times 10^{-8}$ & 0.012 \\
$84.403,-64.000$ & 0.5 & 19.0 & 25 & $3.1 \times 10^{-8}$ & 0.0027 \\
$84.936,-64.100$ & 0.7 & 19.0 & 29 & $4.6 \times 10^{-8}$ & 0.0039 \\
$85.168,-64.400$ & 0.8 & 19.0 & 30 & $7.0 \times 10^{-7}$ & 0.0045 \\
\hline
\end{tabular}

Note: $P$ is the probability of there being $n$ QSO candidates up to magnitude $m_{B, 0}$ in the FH04 catalogue with the probabilities specified by them within a circle of centre $(\alpha, \delta)$ (coordinates $\mathrm{J} 2000)$ and angular radius $R$. $P_{\text {sky }}$ is the probability of finding at least one circle with the small value of $P$ in the whole sky. Cases with $P_{\text {sky }}<0.05$.

complete for galaxies with $m_{B, \text { gal }}<15.5$, redshift $z \leq 0.05$, and an apparent diameter at the $D_{25}$ isophotal level larger than $1 \mathrm{arcmin}$. The catalogue contains a total of 23011 galaxies. From Eq. (1), around all the RC3 galaxies and within a distance of $d$ arcmin and magnitude $m_{B}<m_{B, 0}$, we would expect a number of QSOs:

$N_{\mathrm{RC} 3}\left(m_{B, 0}, d\left(^{\prime}\right)\right) \approx 10^{0.8\left(m_{B, 0}-16.9\right)} d\left(^{\prime}\right) 2$,

which means an average of 70 objects up to 3 arcmin of RC3galaxies with $m_{B} \leq 18$ and 7.6 cases up to 10 arcmin with $m_{B} \leq$ 15.5. Figure 1 shows how FH04 average expectations exceed the expected background counts, and Table 2 shows the very low probability (calculated with Eqs. (2) and (6)) of making both numbers compatible, specially for $d<1^{\prime}$ and $m_{B} \leq 18$. For the highest $d$ values, the probability is somewhat lower due to the incompleteness of the FH04 catalogue rather than to an excess in the number of QSOs.

Among the QSO candidates that are less than 1 arcmin from a RC3 galaxy, we have the examples shown in Fig. 2.

\section{Selection and observation of QSO candidates}

The anomalies in Sect. 2 rely on the probabilities quoted by FH04. To check the reliability of these probabilities, we determined the nature and redshifts of a significant sample of 

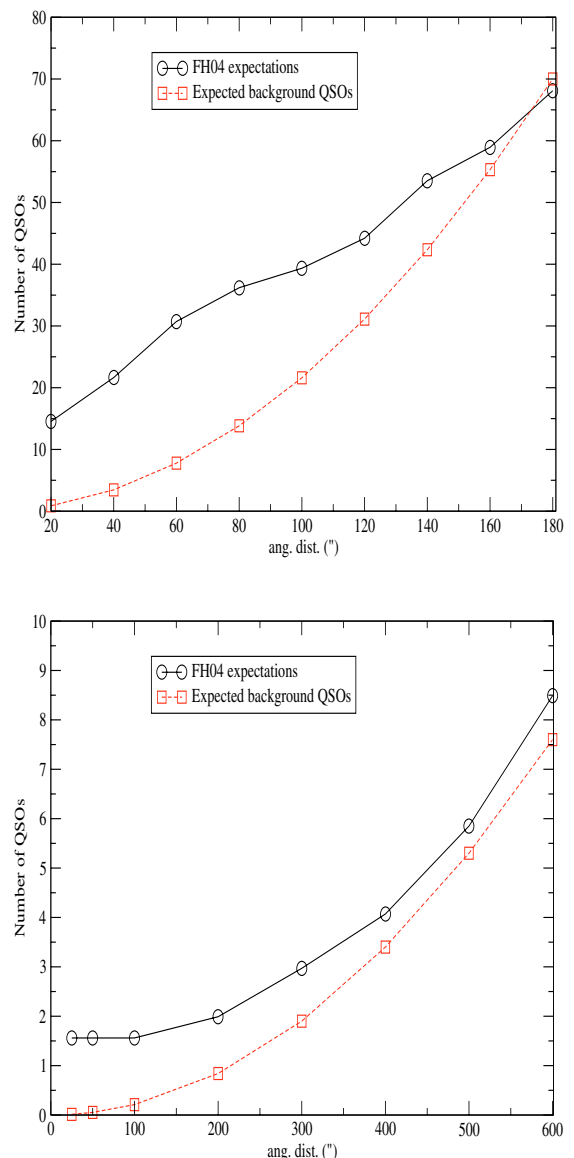

Fig. 1. Cumulative counts of FH4 QSOs (sum of the probabilities given by FH04 to be QSOs) around the complete sample of RC3 galaxies as a function of the maximum distance to a galaxy, in comparison with the expected background QSOs. Up: up to magnitude $m_{B}=18$, Down: up to magnitude $m_{B}=15.5$. QSOs associated to two galaxies count twice with each corresponding distance.

FH04 objects by direct observations. The objects selected follow four criteria:

a) sources up to 3 arcmin from RC3 galaxies with $m_{B} \leq 18$, such as those in Fig. 2;

b) sources up to 10 arcmin from RC3 galaxies with $m_{B} \leq 15.5$;

c) other sources up to 3 arcmin from galaxies with $m_{B} \leq 18$ that do not belong to the RC3 catalogue;

d) sources with $m_{B} \leq 15.5$ (to test the anomalous distribution of this bright sources in several regions of the sky, as shown in Table 1).

In total, we have identified the 41 sources listed in Table 5: 11 from the public database of SIMBAD (data obtained after the publication of FH04) and 30 from our own spectroscopic observations. A double check was made by observing 5 objects already classified in SIMBAD. The columns of Table 5 are as follows. Column 1 gives number of the source. Column 2: equatorial coordinates (J2000) of the optical counterpart identified by FHO4 as a QSO candidate. Column 3: apparent magnitude in the $B$ filter (FH04). Column 4: probability that the source is a QSO according to FH04. Column 5: radio flux at $1.4 \mathrm{GHz}$ (mJy) or flux in X-ray (counts/hour in ROSAT) [FH04]. Column 6: peculiarity of the source: a) sources up to 3 arcmin from RC3 galaxies with $m_{B} \leq 18$; b) sources up to 10 arcmin from RC3 galaxies with $m_{B} \leq 15.5$; c) sources up to 3 arcmin from non RC3 galaxies with $m_{B} \leq 18$; d) sources with $m_{B} \leq 15.5$. In cases a); b); and
Table 2. Probabilities of QSO excesses around RC3 galaxies.

\begin{tabular}{ccccc}
\hline \hline$d\left({ }^{\prime \prime}\right)$ & $m_{B, 0}$ & $n$ & $\left\langle n_{b g}\right\rangle$ & $P_{\mathrm{RC} 3}$ \\
\hline $0-20$ & 18.0 & 25 & 0.86 & $3.5 \times 10^{-7}$ \\
$20-40$ & 18.0 & 12 & 2.6 & 0.027 \\
$40-60$ & 18.0 & 14 & 4.3 & 0.031 \\
$60-80$ & 18.0 & 8 & 6.0 & 0.15 \\
$80-100$ & 18.0 & 5 & 7.8 & 0.042 \\
$100-120$ & 18.0 & 8 & 9.5 & 0.049 \\
$120-140$ & 18.0 & 13 & 11 & 0.10 \\
$140-160$ & 18.0 & 8 & 13 & 0.013 \\
$160-180$ & 18.0 & 13 & 15 & 0.042 \\
$0-25$ & 15.5 & 3 & 0.013 & 0.096 \\
$0-50$ & 15.5 & 3 & 0.053 & 0.11 \\
$0-100$ & 15.5 & 3 & 0.21 & 0.14 \\
$100-200$ & 15.5 & 1 & 0.63 & 0.45 \\
$200-300$ & 15.5 & 2 & 1.1 & 0.32 \\
$300-400$ & 15.5 & 2 & 1.5 & 0.29 \\
$400-500$ & 15.5 & 3 & 1.9 & 0.25 \\
$500-600$ & 15.5 & 3 & 2.3 & 0.22 \\
\hline
\end{tabular}

Note: $P_{R C 3}$ is the probability of having $n$ QSO candidates up to magnitude $m_{B, 0}$ in FH04 catalogue with the probabilities specified by them within a circle centred at a RC3 galaxy and angular distance $d$.

c): the RC3 galaxy (or non-RC3 for case \# 6) from which the source is closest, and the distance (in arcseconds) between the QSO candidate and the galaxy are specified. Column 7: exposure time in seconds (with the superindex ${ }^{n p}$ means non-photometric), date of observation and telescope (CA: 2.15 m CASLEO, San Juan, Argentina; OH: 1.93 m OHP, Haute-Provence, France; IU: 2 m IUCAA, India) or "SIMBAD-database" when the data were publicly available on the SIMBAD web-page. Column 8: spectroscopic identification. Column 9: spectral lines: CIV is at $1549 \AA, \mathrm{CIII}$ is at $1909 \AA, \mathrm{MgII}$ is at $2798 \AA$, NeIII is at $3869 \AA$, $\mathrm{HeI}$ is at $3889 \AA$, CaII is the doublet $\mathrm{H}$ and $\mathrm{K}$ at $3933 / 3970 \AA, \mathrm{H}_{\delta}$ is at $4102 \AA, \mathrm{H}_{\gamma}$ is at $4340 \AA$, HeII is at $4686 \AA, \mathrm{H}_{\beta}$ is at $4861 \AA$, OIIIa is at $4959 \AA$, OIIIb is at $5007 \AA, \mathrm{MgI}$ is at $5180 \AA$, NaI is at $5892 \AA$, OI is at $6300 \AA$, NIIa is at $6548 \AA, \mathrm{H}_{\alpha}$ is at $6562 \AA$, NIIb is at $6584 \AA$, SIIa is at $6717 \AA$, SIIb is at $6731 \AA$; "A:" means absorption, "E:" means emission, superindex $b$ means "broad". Column 10: redshift.

We used the following telescopes and instrumental setups:

- 2.15 m CASLEO, in "El Leoncito" Observatory, San Juan, Argentina. REOSC Ds Spectrograph, grism \# 270, slit width: 2.5". Wavelength range: $3630-7120 \AA$, $3.41 \AA /$ pixel.

- $1.93 \mathrm{~m}$ OHP, Haute-Provence, France. CARELEC Spectrograph, whose grating provides a resolution of $133 \AA / \mathrm{mm}$, slit width: $2^{\prime \prime}$. $1.78 \AA /$ pixel. Wavelength range: 3965-7600 ̊.

- 2 m IUCAA, India. $2 \mathrm{~m} \mathrm{f} / 10$ Cassegrain telescope. IFOSC (IUCAA Faint Object Spectrograph and Camera), f/4.5. Grism FOSC7, wavelength range 3800-6840 A, dispersion $1.39 \AA$ ̊/pixel, slit width: $1.5^{\prime \prime}$.

We followed a standard method of reduction. A summary of the identifications is presented in Table 5. Only 7 of the 30 objects turned out to be QSOs, while the other 23 were stars, HII regions, or galaxies. The spectra of the seven QSOs are plotted in Fig. 3. For the 11 SIMBAD database objects, there were another 5 QSOs, so we found 12 QSOs from a total number of 41 objects observed. 

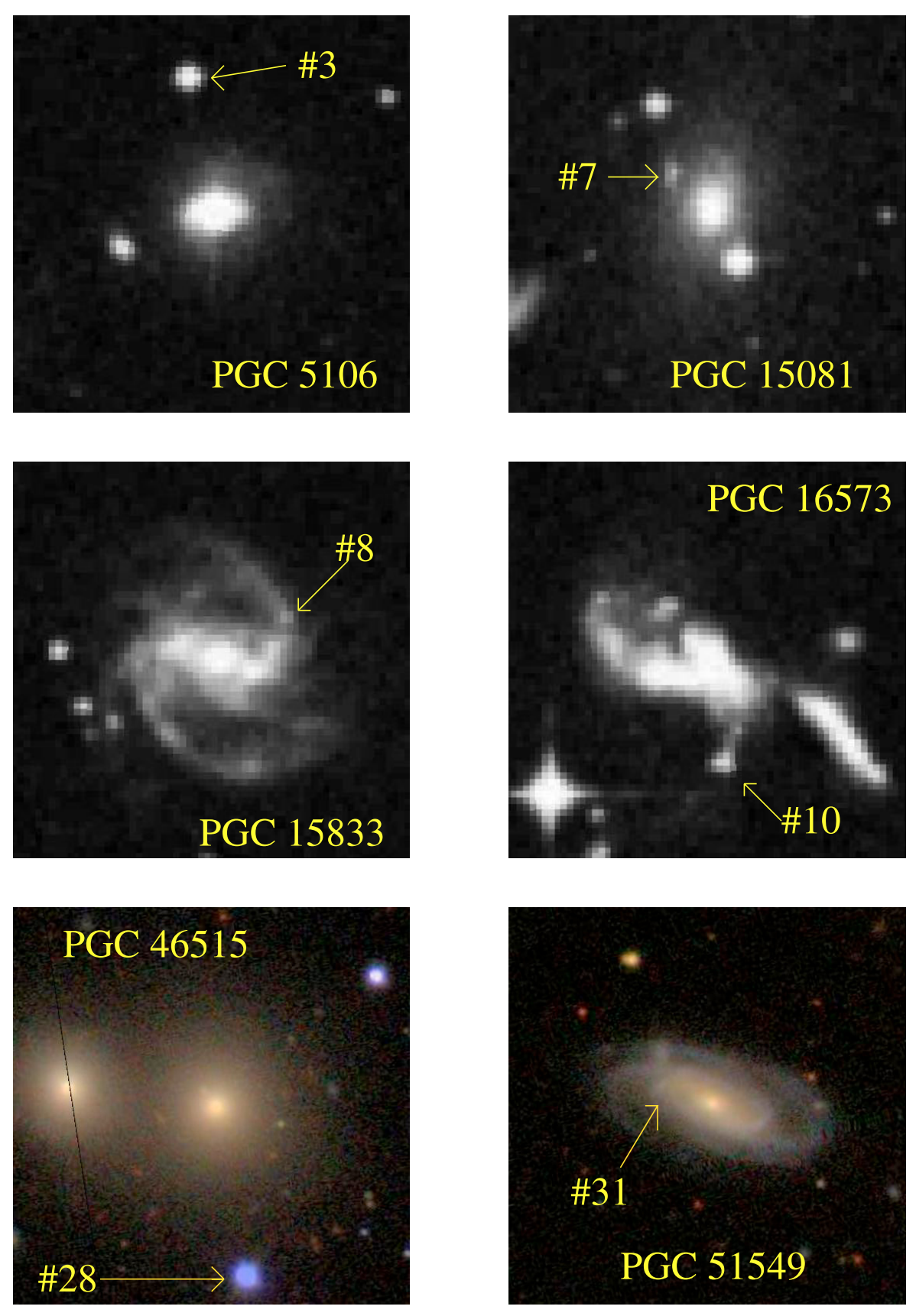

Fig. 2. Some of the QSO candidates of FH04 observed in the present work (number \# as in Table 5). PGC 5106, PGC 15081, PGC 15833, PGC 16573 images from the Digitized Sky Survey, size $108^{\prime \prime} \times 108^{\prime \prime}$. PGC 46515, PGC 51549 images from the Sloan Digital Sky Survey, size $100^{\prime \prime} \times 100^{\prime \prime}$. None of these candidates is a QSO.

\subsection{Notes on some objects}

\#7: this is a Seyfert 2 galaxy at only $13 \mathrm{kpc}$ projected distance from a cD galaxy (PGC 15081) within the same cluster.

$\# 8$,\#24,\#31: these are star-forming regions that were probably considered as QSO candidates on the basis of their blue colours.

\#11,\#16, \#20,\#32: white dwarfs are also usually misidentified as QSO candidates because of their blue colours.

\#15: this is a cold M5 star with emission lines presumably caused by photospheric activity, which gives strong X-ray emission.

The rest of the non-QSO objects are other types of stars. The number of contaminating objects in case a) (up to magnitude 18 within $180 \mathrm{deg}^{2}$ ) or b) (up to magnitude 15.5 within $2000 \mathrm{deg}^{2}$ ) is not surprising since the number of stars in high galactic latitude fields is $\sim 10^{5}-10^{6}$ (Robin et al. 2003) for both cases a) and $\mathrm{b}$ ), and among them a fraction $\sim 10^{-4}-10^{-5}$ might be white dwarfs or stars with some activity or feature that make them susceptible to being detected in radio or X-ray.

\section{The reliability of $\mathrm{FHO4}$ probabilities}

\subsection{As a function of the apparent magnitude}

Given the observed number of QSOs that have different magnitudes with the a priori probabilities of FH04 (4th column of Table 5), we can calculate $P(\leq m)$ with Eq. (4), the probability that FH04 expectations are correct given that we have detected a number of $\leq m$ QSOs among the $n$ candidates. They are given in Table 3. Clearly, for magnitudes $m_{B}<\approx 17$, the a priori probabilities given by FH04 are overestimated. However, for $m_{B}>\approx 17$ our results are compatible with the probabilities given by FH04. Figure 4 and Table 3 show the expected and the observed average probabilities of the FH04 candidates being QSOs. 

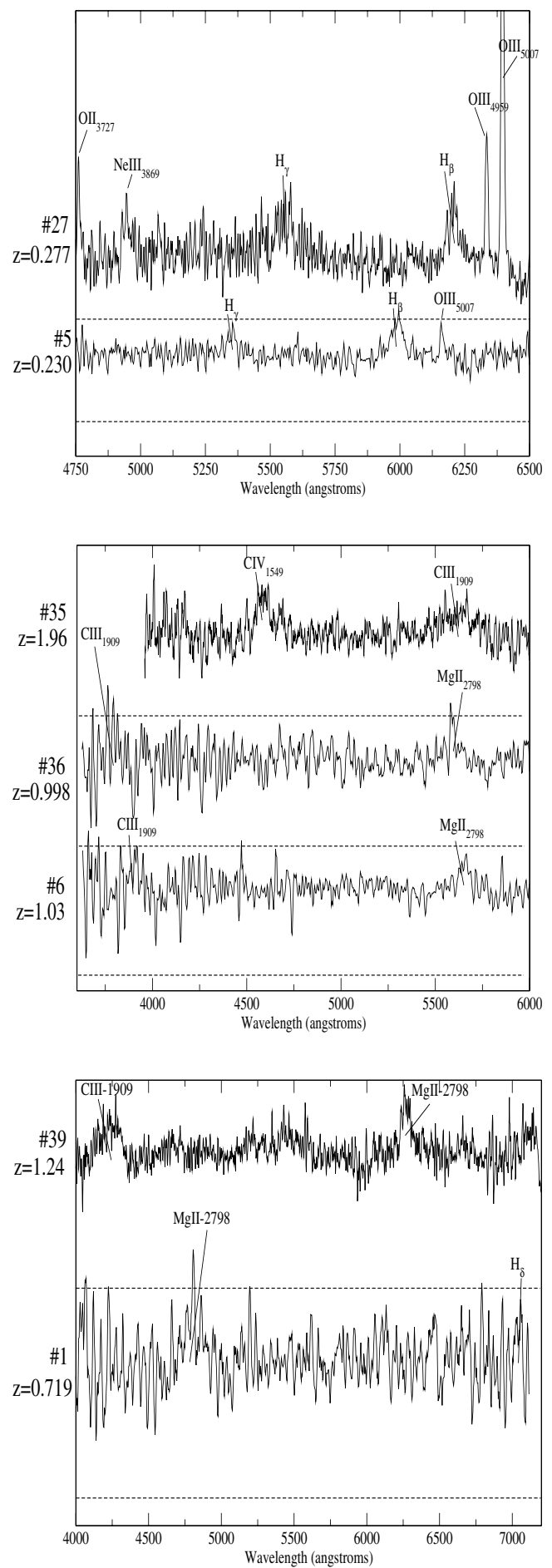

Fig. 3. Continuum normalized spectra of the seven objects classified as QSOs from a total of 30 FH04 candidates. Dashed lines stand for zero flux.

Given that none of the 13 QSO candidates with $m_{B} \leq 15.1$ are in fact QSOs, this means that the excesses of Table 1 with $m_{B} \leq 15$ are not real QSO excesses, so there is no incompatibility with background expectations.

\subsection{As a function of the distance to an RC3 galaxy}

For the subsample a) of Table 5, there are 26 objects with $m_{B} \leq 18$ at distances less than $180^{\prime \prime}$ from an RC3 galaxy,
Table 3. Probabilities that FHO4 expectations are correct as a function of the magnitude.

\begin{tabular}{ccccc}
\hline \hline$m_{B}$ & $n$ & $m$ & $m_{\mathrm{FH} 04}$ & $P(\leq m)$ \\
\hline $13.4-14.4$ & 6 & 0 & $3.9_{-2.5}^{+1.2}$ & $1.1 \times 10^{-4}$ \\
$14.7-15.1$ & 7 & 0 & $4.7_{-2.5}^{+1.3}$ & $5.2 \times 10^{-5}$ \\
$15.2-16.5$ & 7 & 2 & $5.5_{-2.3}^{+1.2}$ & $2.4 \times 10^{-3}$ \\
$16.7-17.2$ & 7 & 2 & $5.3_{-2.6}^{+1.3}$ & 0.011 \\
$17.3-17.7$ & 7 & 4 & $5.1_{-2.4}^{+1.2}$ & 0.23 \\
$17.8-18.0$ & 7 & 4 & $5.5_{-2.3}^{+1.2}$ & 0.14 \\
\hline
\end{tabular}

Note: Column 2: $n$ : number of FH04 QSO candidates observed. Column 3: $m$ number of these which are identified spectroscopically as QSOs. Columm 4: expected number of QSOs according to FH04 ( \pm ; 95\% C.L.). Column 5: $P(\leq m)$, probability that FH04 expectations are correct given that we have detected a number $\leq m$ of QSOs among the $n$ candidates.

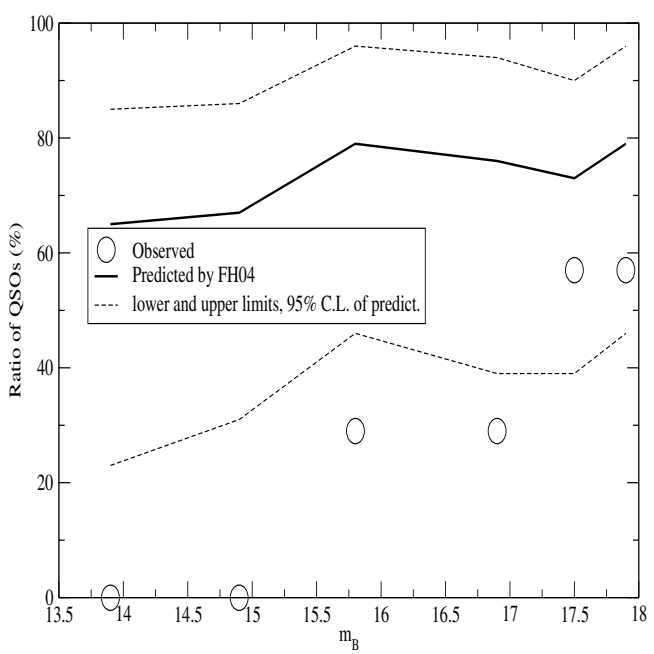

Fig. 4. Observed ratio of QSOs as a function of magnitude among the $41 \mathrm{FH} 04$ candidates with spectroscopic identification given in this paper. Comparison with predictions of FH04 (limits within dashed line are 95\% C.L.).

36 cases if we count the repetitions ${ }^{3}$. Table 4 gives the probability $P(\leq m)$ that FH04 expectations are correct given that we detected a number of $\leq m$ QSOs among the $n$ candidates, as a function of the distance of the RC3 galaxy. Figure 5 and Table 4 show the expected and the observed average probability of the FHO4 candidates being QSOs. We conclude that the FH04 probabilities are highly overestimated in the regions within $\sim 1$ arcmin from an RC3 galaxy. There is also overestimation at distances $\sim 2-3$ arcmin.

This result solves the second anomaly. Since only 1 object among 11 candidates at distances less than 1 arcmin is really a QSO, the probabilities given by $\mathrm{FH} 04$ are not valid, and the announced excess announced in Sect. 2.2 is not supported.

\section{Discussion and conclusions}

In FH04 there are 88 different objects (106 cases with repetitions) with $m_{B} \leq 18$ within a radius of 3 arcmin of RC3 galaxies. Of these 88 objects, there are spectroscopic observations of the

\footnotetext{
3 There are some cases of two RC3 galaxies projected very close in the sky. For these cases QSOs projected near such galaxies are counted twice (we will refer to these cases as "cases with repetition"), so this raises the number of QSO/galaxy associations to 36 .
} 
Table 4. Probabilities that FHO4 expectations are correct as a function of the distance to RC3 galaxies.

\begin{tabular}{ccccc}
\hline \hline$d\left({ }^{\prime \prime}\right)$ & $n$ & $m$ & $m_{\mathrm{FH} 04}$ & $P(\leq m)$ \\
\hline $0-30$ & 4 & 0 & $2.9_{-2.0}^{+0.9}$ & $4.2 \times 10^{-3}$ \\
$30-60$ & 7 & 1 & $5.2_{-2.6}^{+1.3}$ & $1.1 \times 10^{-3}$ \\
$60-90$ & 3 & 1 & $2.7_{-1.6}^{+0.2}$ & 0.022 \\
$90-120$ & 5 & 3 & $3.0_{-2.2}^{+1.2}$ & 0.68 \\
$120-150$ & 8 & 3 & $6.1_{-2.6}^{+1.4}$ & 0.015 \\
$150-180$ & 9 & 2 & $6.6_{-2.3}^{+1.2}$ & $8.8 \times 10^{-4}$ \\
\hline
\end{tabular}

Note: Column 1: range of distances from an RC3 galaxy. Column 2: $n$ : number of FH04 QSO candidates observed. Column 3: $m$ number of these which are identified spectroscopically as QSOs. Columm 4: expected number of QSOs according to FH04 ( \pm ; 95\% C.L.). Column 5: $P(\leq m)$, probability that FH04 expectations are correct given that we have detected a number $\leq m$ of QSOs among the $n$ candidates.

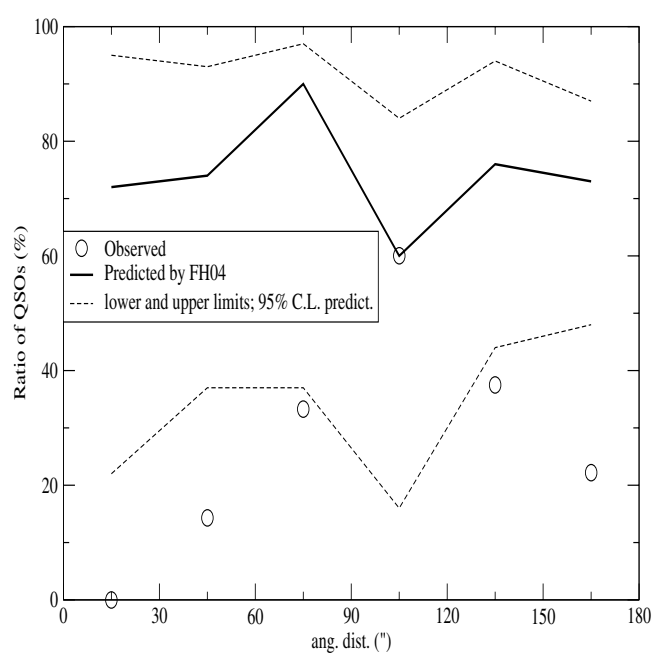

Fig. 5. Observed ratio of QSOs as a function of distance to an RC3 galaxy among the $36 \mathrm{FH} 04$ candidates (including repetitions, i.e. objects associated with more than one galaxy) with spectroscopic identification given in this paper. Comparison with the predictions of FH04 (limits within dashed line are 95\% C.L.).

optical counterparts in 26 cases (36 cases including repetitions), and 10 of these (the same number with repetitions) are in fact QSOs (while the 16 other objects (26 with repetitions) are stars, galaxies, or HII regions). The average expected number of background cases around all RC3 cases should be $\approx 70$ (Sect. 2.2) and, in our subsample, should be $\approx \frac{36}{106} \times 70=24$ cases, which is higher than the value derived from the observations. This stems from the incompleteness of the FH04 catalogue.

Within a radius of 10 arcmin of RC3-galaxies, there are 14 objects (no repetitions) with $m_{B} \leq 15.5$, of which five $(36 \%$ of the sample) were observed and only one (\# 5 with $m_{B}=15.4$ ) turned out a QSO. The average expected number of cases around all RC3 galaxies is 7.6 (Sect. 2.2), and in our subsample should be $\frac{5}{14} \times 7.6=2.7$ cases. This result agrees with expectations from a background distribution of QSOs.

Limiting the study to RC3 galaxies by $m_{B \text {, gal }} \leq 14.5$, $m_{B \text {, gal }} \leq 13.5$, and $m_{B \text {, gal }} \leq 12.5$, the number of galaxies is reduced to $7,675 / 2,566 / 883$. For the subsample with $m_{b} \leq 18$, $d \leq 3^{\prime}$ we obtained 5/2/2 QSOs to be compared with the expected 7.8/2.7/0.92. For the subsample with $m_{b} \leq 15.5, d \leq 10^{\prime}$, we obtained $1 / 0 / 0$ to be compared with $0.50 / 0.17 / 0.06$. There

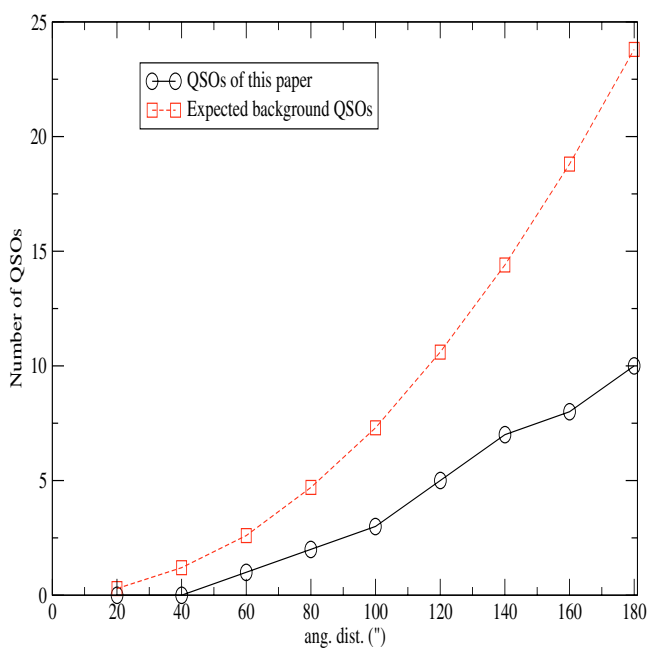

Fig. 6. Cumulative counts of QSOs of this paper up to magnitude $m_{B}=$ 18 [34\% (36/106) of the QSO/RC3-galaxy pairs] as a function of the maximum distance to the galaxy. QSOs associated with two galaxies count twice with each corresponding distance.

is no statistical inconsistency with the claim that all QSOs are background QSOs rather than being associated with the galaxies.

As for the distribution of the QSOs with magnitude or distance from RC3 galaxies, as pointed out in Sect. 4, there is no longer any inconsistency with background predictions: almost all the confirmed QSOs in our subsample are faint (all of them with magnitudes $m_{B} \geq 17.0$, except \#5) and distant enough from the centres of RC3 galaxies to be compatible with background expectations. See Fig. 6 for the distance distribution and note how the observed QSOs are fewer than the background expectations for all distances. Some of the most troublesome candidates, such as those shown in Fig. 2, were in the end confirmed to be objects different from QSOs.

The only QSO with $m_{B} \leq 15.5$ (\# 5) within $10^{\prime}$ from an RC3 galaxy is associated with NGC 1136, which was previously reported as a galaxy with peculiar associations (Arp 1981). Objects claimed by Arp to be anomalous are much fewer than 23011 RC3 galaxies. They are $\sim 10^{2}$, so the probability of finding a QSO with $m_{B} \leq 15.5$ within $10^{\prime}$ from some of them is $P \sim 0.06$. It is not few enough to claim it separately from other facts as a possible anomalous case, but a fact to be added in the discussion of the case of NGC 1136.

For the object \# 6, now confirmed to be a QSO with $m_{B}=$ 16.5 at only $30^{\prime \prime}$ from PHL 1459 (a galaxy with $m_{B}=17.2$ and $\mathrm{X}$-ray emission), we are not within the statistics of RC3 galaxies. There are $\approx 4.3 \times 10^{5}$ galaxies in the whole sky up to the magnitude $m_{B}=17.2$ (Metcalfe et al. 1991), so, using Eq. (1), the expected number of cases like this within a 30 -arcsec distance of these galaxies is 2.2 . The discovery of only one case is not enough to claim any statistical anomaly. If we take into account the peculiarity that PHL 1459 is an X-ray source and has $\approx 120$ counts/h in ROSAT (Voges et al. 1999, 2000), the probability is somewhat lower. There are $\sim 0.4$ galaxies $/ \mathrm{deg}^{2}$ up to this X-ray flux (Voges et al. 1999; Zickgraf et al. 2003), i.e. around 16000 galaxies like this in the whole sky. The probability of finding a QSO with $m_{B}=16.5$ within $30^{\prime \prime}$ of one of them is 0.09 ; not low enough to discard a background projection coincidence.

Summing up, within the subsample of 41 objects from FH04, we have not found any statistical anomaly with respect to the expectation of QSOs as background sources. Since we observed a representative subsample of the most controversial cases that 


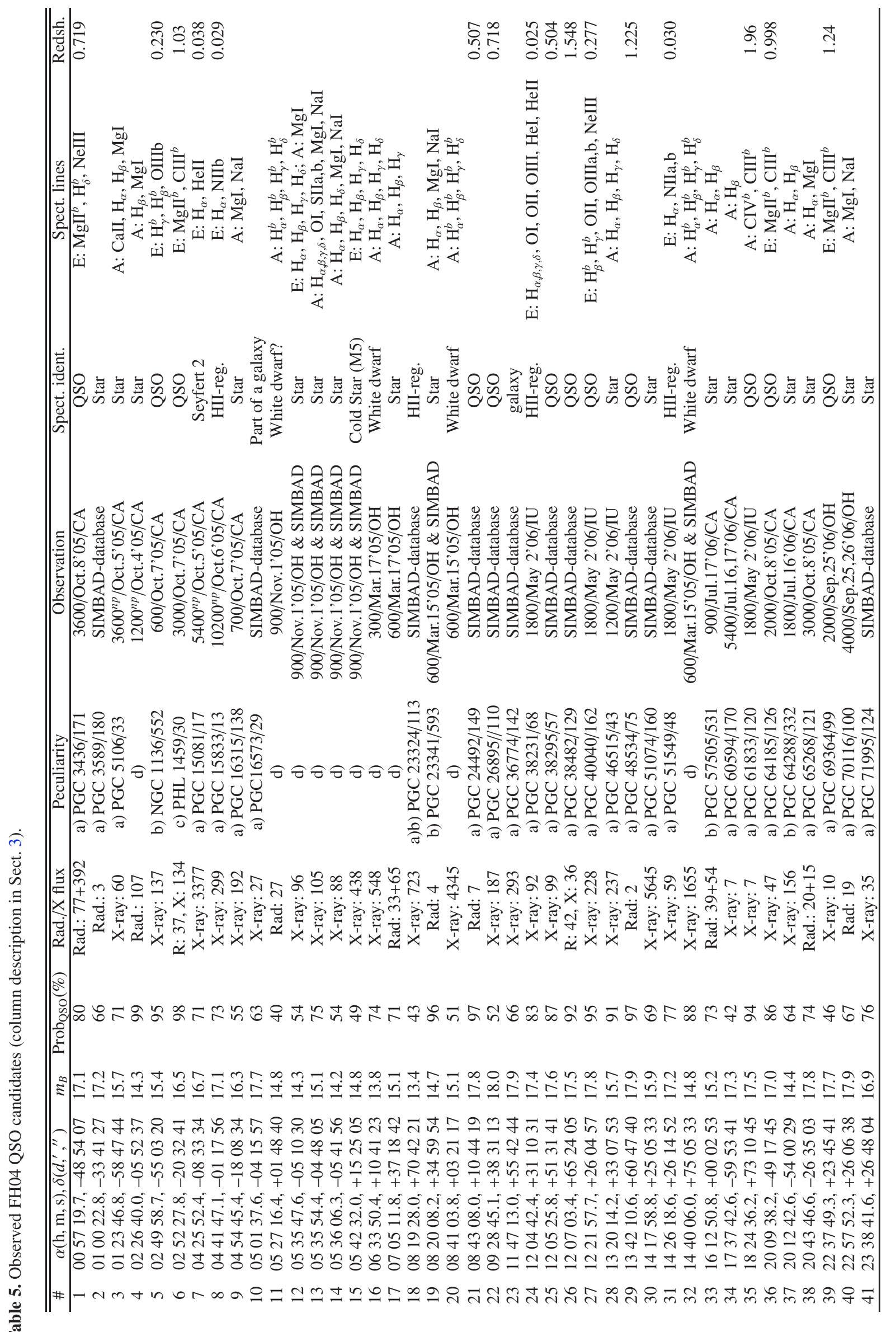


could in principle present some excesses of QSOs, we think that there should be in principle no reason to expect statistical anomalies in the FH04 catalogue, which is anyway a useful database for looking for QSOs in regions not covered by other surveys, such as SDSS or $2 \mathrm{dF}$, or for deeper magnitudes. Nevertheless, there are still anomalies in the QSO distributions presented with some other catalogues (e.g. Arp 1998; Burbidge 2001) that require further attention. Further rigorous statistical analysis like those presented here and in López-Corredoira \& Gutiérrez $(2006 a, b)$ are necessary.

Acknowledgements. Thanks are given to Rubén J. Díaz (Gemini Observatory) for providing telescope time at $2.15 \mathrm{~m}$ CASLEO, to Eric Flesch for his comments and criticism on a draft of this paper, to the referee Mira Veron for her helpful comments for improving it, to T. J. Mahoney (IAC) and Joli Adams (A\&A) for proofreading the paper.

Based on observations made with the telescopes: $2.15 \mathrm{~m}$ CASLEO in the observatory of "El Leoncito", San Juan, Argentina; 1.93 m OHP (these observations were funded by the Optical Infrared Coordination network, OPTICON, a major international collaboration supported by the Research Infrastructures Programme of the European Commission Sixth Framework Programme), Haute-Provence, France; and 2 m IUCAA (Pune, India).

This research has made use of the SIMBAD database, operated at CDS, Strasbourg, France.

The Digitized Sky Surveys were produced at the Space Telescope Science Institute under US Government grant NAG W-2166. The images of these surveys are based on photographic data obtained using the Oschin Schmidt Telescope on Palomar Mountain and the UK Schmidt Telescope. The plates were processed into the present compressed digital form with the permission of these institutions.

Funding for the creation and distribution of the SDSS Archive has been provided by the Alfred P. Sloan Foundation, the Participating Institutions, the National Aeronautics and Space Administration, the National Science Foundation, the US Department of Energy, the Japanese Monbukagakusho, and the Max Planck Society. The SDSS Web site is http://www.sdss.org/. The SDSS is managed by the Astrophysical Research Consortium (ARC) for the Participating Institutions. The Participating
Institutions are The University of Chicago, Fermilab, the Institute for Advanced Study, the Japan Participation Group, The Johns Hopkins University, the Korean Scientist Group, Los Alamos National Laboratory, the Max-Planck-Institute for Astronomy (MPIA), the Max-Planck-Institute for Astrophysics (MPA), New Mexico State University, University of Pittsburgh, University of Portsmouth, Princeton University, the United States Naval Observatory, and the University of Washington.

The first author (M.L.C.) was supported by the Ramón y Cajal Programme of the Spanish Science Ministry.

\section{References}

Adelman-McCarthy, J. K., Agüeros, M. A., Allam, S. S., et al. 2007, ApJS, 172, 634 Arp, H. C. 1981, ApJS, 46, 75

Arp, H. C. 1998, Seeing Red, Apeiron, Montreal

Burbidge, G. R. 2001, PASP, 113, 899

Croom, S. M., Smith, R. J., Boyle, B. J., et al. 2004, MNRAS, 349, 1397

de Vaucouleurs, G., de Vaucouleurs, A., Corwin, H. G., et al. 1991, Third Reference Catalog of Bright Galaxies (New York: Springer) (RC3)

Dobrzycki, A., Eyer, L., Stanek, K. Z., \& Macri, L. M. 2005, A\&A, 442, 495

Flesch, E., \& Hardcastle, M. J. 2004, A\&A, 427, 387 (FH04)

Haberl, F., \& Pietsch, W. 1999, A\&AS, 139, 277

Kahabka, P. 2002, A\&A, 388, 100

López-Corredoira, M., \& Gutiérrez, C. M. 2006a, A\&A, 454, 77

López-Corredoira, M., \& Gutiérrez, C. M. 2006b, in First Crisis in Cosmology Conference, ed. E. J. Lerner, \& J. B. Almeida, American Institute of Physics, AIP Conf. Proc., 822, 75

Metcalfe, N., Shanks, T., Fong, R., \& Jones, L. R. 1991, MNRAS, 249, 498

Pietsch, W., \& Kahabka, P. 1993, in New Aspects of Magellanic Clouds Research, ed. B. Baschek, G. Klare, \& J. Lequeux, Lecture Notes in Physics, 416, 59

Robin, A. C., Reylé, C., Derrière, S., \& Picaud, S. 2003, A\&A, 409, 523

Voges, W., Aschenbach, B., Boller, Th., et al. 1999, A\&A, 349, 389

Voges, W., Aschenbach, B., Boller, Th., et al. 2000, IAU Circ., 7432

Zickgraf, F. J., Engels, D., Hagen, H. J., Reimers, D., \& Voges, W. 2003, A\&A, 406, 535 